\title{
Optimum values of electrical circuit for energy harvesting using a beam and piezoelectric elements
}

\author{
Keisuke YAMADA*, Junichi KURATA*, Hideo UTSUNO* and Yoshihiro MURAKAMI* \\ ${ }^{*}$ Faculty of Engineering Science, Kansai University, \\ 3-3-35 Yamate-cho, Suita-shi, Osaka 564-8680, Japan \\ E-mail: yamadak@kansai-u.ac.jp
}

Received: 13 January 2017; Revised: 3 April 2017; Accepted: 11 May 2017

\begin{abstract}
This paper describes optimum values of an electrical circuit for energy harvesting using a beam and piezoelectric elements. Energy harvesting techniques recover energy from the environment. We target the energy harvesting methods that extract energy from mechanical vibration systems in this research. Vibration energy is usually extracted through electrical circuits using electromechanical transducers such as DC motors and piezoelectric elements. In order to extract energy effectively, these electrical circuits and additional vibration systems must be optimally tuned. The optimum values of an electrical circuit and additional vibration system can be derived based on impedance matching when a vibration source includes only a single excitation frequency; however, in certain cases, vibration sources include several excitation frequencies. The optimum values derived based on impedance matching are not optimum when the vibration source includes several excitation frequencies. Therefore, we additionally investigated the case of random excitation as a representative of such cases in this research. The optimum values for the case of random excitation are also effective for the cases of free vibration including impulse excitation. The governing equations were formulated using equivalent mechanical models in this research. The combination of a beam and a piezoelectric element was used as the piezoelectric energy harvester because the thorough formulation of the governing equations for this configuration was not found in the research literature. The optimum values of the electrical circuit and additional vibration system were derived using the governing equations. The effectiveness of the optimum values was verified through numerical calculations.
\end{abstract}

Key words : Energy harvesting, Piezoelectric element, Beam, Bending vibration, Random excitation, Impedance matching

\section{Introduction}

Researchers have studied many types of energy harvesting techniques to date. While there are many types of energy harvesting techniques, we target the energy harvesting techniques that extract energy from mechanical vibration systems in this research. There are several methods to transform vibration energy into electrical energy. We specifically address the energy transformation using the piezoelectric effect in this paper. However, the energy transformation using the electromagnetic induction is additionally described in this paper for comparison and confirmation of basics. To extract energy from mechanical vibration systems, additional vibration systems are usually installed on vibration sources. In addition, DC motors, electromagnets, or piezoelectric elements are installed into the additional vibration systems. These DC motors, electromagnets, and piezoelectric elements are used as electromechanical transducers. Vibration energy is harvested as electrical energy using a coupled load electrical circuit. The additional vibration systems and electrical circuits should be tuned optimally in order to enhance the efficiency of energy harvesting. The optimum values of the additional vibration system and electrical circuit can be derived based on impedance matching when vibration sources include only a single excitation frequency (Kong et al., 2010). However, in practice, this condition is not satisfied in many cases because vibration sources usually include several excitation frequencies. As solutions for this problem, several types of wideband energy harvesting methods have been proposed (Litak et al., 
2010, Masuda et al., 2013, Yamamoto et al., 2015, Yang et al., 2009). A summary of these types of wideband energy harvesting methods was reported in the review article by Tang et al. (2010). However, these methods are still in the research phase because of their complex mechanisms and requirement of control. The control has the disadvantage of requiring driving energy. Therefore, as a simpler method, the optimum values of the electrical circuit for the case of random excitation using white noise has been theoretically derived as a representative of the cases where the vibration source includes several excitation frequencies (Adhikari et al., 2009, Halvorsen, 2008, Hendijanizadeh, 2014, Tang and Zuo, 2012). The optimum values for the case of random excitation are effective for the case of the impulse excitation because the frequency spectrum of the impulse excitation is proportional to that of white noise.

When a DC motor or electromagnet is used as an electromechanical transducer, only a resistor is coupled to the DC motor or electromagnet because the inductance of a DC motor or electromagnet is usually negligible. In contrast, the capacitance of a piezoelectric element is not negligible. However, an additional electrical circuit is not usually coupled to a resistor even if a piezoelectric element is used. In the case of passive vibration suppression using piezoelectric elements, it is a well-known fact that an inductor-resistor (LR) circuit is more effective than a resistive circuit. This fact indicates that an inductor should be added to the resistor to increase the energy harvesting efficiency in accordance with the conditions (Renno et al., 2009, Adhikari et al., 2009). The reason that an LR circuit has been seldom used in energy harvesting is that an actual inductor becomes too large for practical applications. In the case of passive vibration suppression, a generalized impedance converter is usually used instead of an actual inductor (Yamada et al., 2010). A generalized impedance converter requires two operational amplifiers. Therefore, this method has been little-used in energy harvesting. However, we proposed the method to shrink the size of an actual inductor using a multi-layered piezoelectric element in the research of passive vibration suppression (Yamada, 2017). This method solves the problem of size of an actual inductor, and allows the optimum values of the LR circuit to be derived. A series LR circuit is used in this research because only the parallel LR circuit has been used in the previous researches (Renno et al., 2009, Adhikari et al., 2009). In passive vibration suppression using piezoelectric elements, there is little difference between the series and parallel LR circuits in terms of performance; however, it is well-known fact that the optimum values of the resistances are much different between them (Yamada el al., 2010). Therefore, the series LR circuit can be an option depending on the value of the resistance of the coupled load electrical circuit.

Many types of load electrical circuits have been intensively studied to enhance the efficiency of energy harvesting from a practical perspective (Caliò et al., 2014, Ottman et al., 2002, 2003, Guyomar et al., 2005). Many researchers have shown an interest in increasing harvested energy of the experimental devices. However, there is no sense at worst if amounts of harvested energy are shown without providing theoretical maximum values. In order to solve this problem, several normalized indices have been defined to evaluate the efficiency (Mitcheson et al., 2004, 2008, Roundy, 2005). However, the theoretical analysis of the previous researches appears to be insufficient. The case of a single excitation frequency has been theoretically analyzed by Kong et al. (2010), Renaud et al. (2008), Renno et al. (2009), and Stephen (2006). In addition, the case of random excitation has been relatively recently studied by Adhikari et al. (2009), Halvorsen (2008), Hendijanizadeh (2014), and Tang and Zuo (2012). However, there are still two problems: one is that the piezoelectric energy harvester using bending vibration of beams has not been theoretically analyzed, and the other is that piezoelectric energy harvesters using a series LR circuit have not been investigated as abovementioned. The objective of this research is to solve these problems, specifically to derive and provide the versatile optimum values of the additional vibration system and series LR circuit, and the value of harvested energy based purely on theoretical analysis. Therefore, we do not go into the details of practical electrical circuits in this research. The part of the electrical circuit that extracts or consumes energy is simply modeled as a resistor in this research. This modeling is commonly used regardless of whether electromagnetic induction or the piezoelectric effect is used. In certain cases, a capacitor is used for storing energy; however, this case is not described in this paper.

In this research, the governing equations are theoretically derived using the typical energy harvesting models. The combination of a beam and a piezoelectric element is used as the piezoelectric energy harvester because the thorough theoretical formulation for this case was not found in the research literature. The optimum values of the series LR circuit and additional vibration system are formulated for both cases of the harmonic excitation and random excitation using white noise. The effectiveness of the optimum values is verified through numerical calculation. 


\section{Dynamic model and governing equations}

The part of the electrical circuit that extracts or consumes energy is modeled as a resistor with resistance $R$ in this research. The vibration source is modeled as the base excitation with displacement $x_{0}$, which is effective under the condition that the mechanical impedance of the vibration source is significantly larger than the mechanical impedance of the additional vibration system. Under this condition, the vibration characteristics of the additional vibration system have little influence on the vibration source. In fact, the acceleration $\ddot{x}_{0}$ is determined by the vibration characteristics and excitation condition of the vibration source; however, for simplicity, only the case where the acceleration $\ddot{x}_{0}$ is given by white noise is investigated in this research. As mentioned Section 1, the energy harvester using the electromagnetic induction is also briefly described for comparison and confirmation of basics.

\subsection{Dynamic model and governing equation for energy harvesting using electromagnetic induction}

The analytical model for the energy harvester using a DC motor is shown in Fig. 1(a). Here, $m_{1}, d_{1}$, and $k_{1}$ are the mass of the mass point, damping coefficient of the dashpot, and spring constant of the spring, respectively, $x_{1}$ is the displacement of the additional one degree of freedom vibration system, and $\dot{q}$ is the electrical current. Obviously, an electrical magnet can be used instead of the DC motor. The equation of motion is given as

$$
m_{1} \ddot{x}_{1}+d_{1}\left(\dot{x}_{1}-\dot{x}_{0}\right)+k_{1}\left(x_{1}-x_{0}\right)+K_{T E} \dot{q}=0
$$

where $K_{T E}$ is the torque constant of the DC motor or electrical magnet. The torque constant is equal to the back-EMF constant of the DC motor. When a DC motor is used, the mechanism that converts linear motion into rotational motion is required. However, for simplicity, the effect of such a mechanism was not considered in Eq. (1). The inductance is not considered in this research because the absolute value of the electrical impedance of the inductance is usually much smaller than that of the internal resistance of a DC motor or electromagnet. The internal resistance is not negligible (Stephen, 2006); however, the internal resistance is not considered in this research because the objective of describing the energy harvesting using electromagnetic induction is to obtain the essential knowledge. The circuit equation is expressed as

$$
R \dot{q}=K_{T E}\left(\dot{x}_{1}-\dot{x}_{0}\right) .
$$

From Eqs. (1) and (2), the equation of motion is given as

$$
\begin{aligned}
& m_{1} \ddot{x}_{1}+\left(d_{1}+d_{2}\right)\left(\dot{x}_{1}-\dot{x}_{0}\right)+k_{1}\left(x_{1}-x_{0}\right)=0, \\
& d_{2}=\frac{K_{T E}^{2}}{R},
\end{aligned}
$$

where $d_{2}$ is the equivalent damping coefficient of the dashpot that corresponds to the resistor. From Eq. (3), the equivalent mechanical model of the analytical model is shown in Fig. 1(b). The cylinder is fixed in the space in Fig. 1(b). The volume in the cylinder is constant, and the pressure is uniform in the cylinder. The areas of three pistons are equal, and the masses of the pistons and medium in the cylinder are negligible. The cylinder and pistons transmit the relative displacement to the dashpot that corresponds to the resistor. In fact, the equivalent mechanical model shown in Fig. 1(b) can be transformed into the simpler model shown in Fig. 1(c); however, the model shown in Fig. 1(b) is
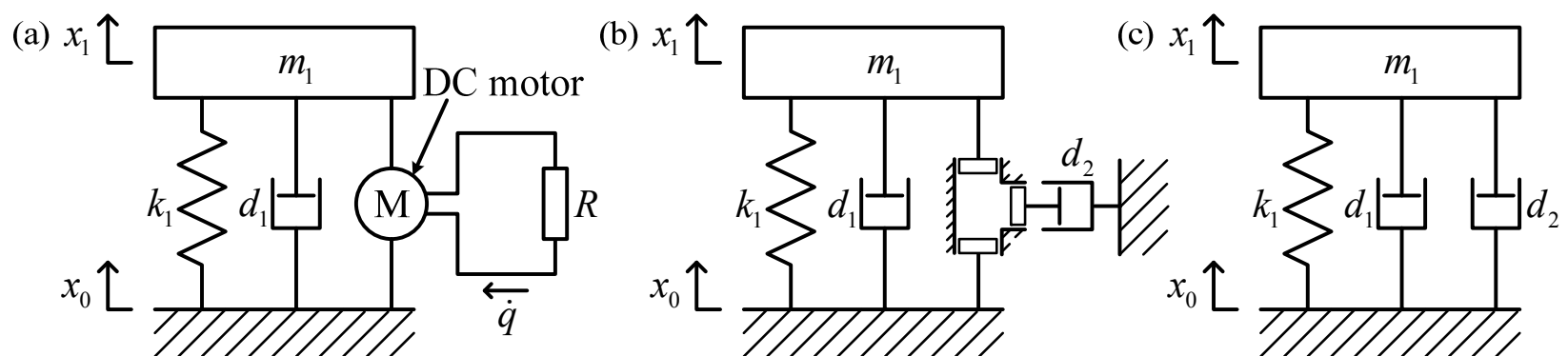

Fig. 1 Analytical model and its equivalent mechanical models of the energy harvester using a DC motor. 
useful when the impedance matching is considered in Section 3.

\subsection{Dynamic model and governing equations for energy harvesting using piezoelectric effect}

The analytical model for the energy harvester using a beam and a piezoelectric element is shown in Fig. 2(a). A plate-type piezoelectric element is used in this analytical model. The additional vibration system consists of a beam and a mass in this analytical model. A series LR circuit is coupled to the piezoelectric element in Fig. 2(a). When a resistive circuit is coupled to the piezoelectric element, the inductance $L$ should be zero in Fig. 2(a). In this section, firstly, the equivalent mechanical model of the piezoelectric element and electrical circuit is derived using the analytical model shown in Fig. 3(a). In other word, the beam is not considered in this first step. Secondly, the governing equations with considering the beam are formulated using the modal analysis and equivalent mechanical model of the piezoelectric element and electrical circuit. Finally, the equivalent mechanical model of the whole system is obtained.

The piezoelectric constitutive equations of the piezoelectric element shown in Fig. 3(a) are expressed as

$$
\begin{aligned}
& S_{1}=\frac{1}{E_{p}} T_{1}+d_{31} E_{3}, \\
& D_{3}=d_{31} T_{1}+\varepsilon_{33}^{T} E_{3},
\end{aligned}
$$

where $S_{1}$ is the strain, $T_{1}$ is the stress, $E_{3}$ is the electrical field, $D_{3}$ is the electric flux density, $E_{p}$ is the Young's modulus, $d_{31}$ is the piezoelectric constant, and $\varepsilon_{33}^{T}$ is the electrical permittivity of the piezoelectric element. The subscript 1 denotes the longitudinal direction, 3 and 33 denote the thickness direction, and 31 denotes that the electric flux density is the thickness direction and the strain is the longitudinal direction. The superscript $T$ denotes the value that is obtained under constant stress. For simplicity, the effect of the bonding layer, strain in the width direction, and the mass of the piezoelectric element are neglected. Equations (5) and (6) can be respectively transformed into the following equations:

$$
\begin{aligned}
& x_{p}=\frac{1}{k_{m}} f_{p}+\frac{\theta_{p}}{k_{m}} V_{p}, \\
& q=\frac{\theta_{p}}{k_{m}} f_{p}+C_{p}^{T} V_{p}=\theta_{p} x_{p}+C_{p}^{S} V_{p}, \\
& x_{p}=l_{p} S_{1}, f_{p}=w_{p} t_{p} T_{1}, V_{p}=t_{p} E_{3}, q=l_{p} w_{p} D_{3}, k_{m}=\frac{E_{p} w_{p} t_{p}}{l_{p}}, \theta_{p}=d_{31} E_{p} w_{p}, \\
& C_{p}^{T}=\frac{\varepsilon_{33}^{T} l_{p} w_{p}}{t_{p}}, C_{p}^{S}=C_{p}^{T}-\frac{\theta_{p}^{2}}{k_{m}},
\end{aligned}
$$

where $x_{p}$ is the displacement, $f_{p}$ is the force, $V_{p}$ is the voltage, $q$ is the charge, and $l_{p}, w_{p}$, and $t_{p}$ are the length, width, and thickness of the piezoelectric element, respectively. The superscript $S$ denotes the value that is obtained under constant strain. Note that the same symbol used in Section 2.1 is used for the charge $q$; however, they have no relationship.

When a series LR circuit is coupled to the piezoelectric element, the voltage $V_{p}$ is expressed as

(a)

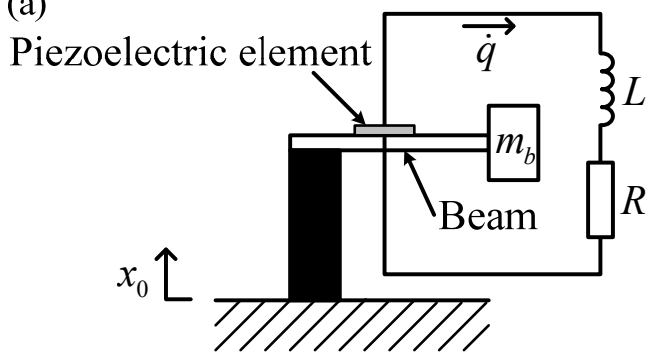

(b)

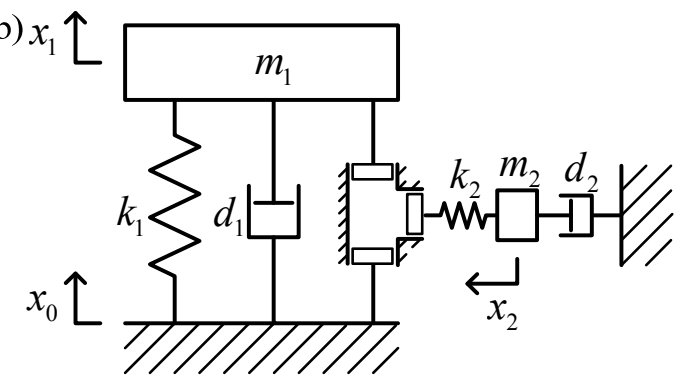

Fig. 2 Analytical model and its equivalent mechanical model of the energy harvester using a piezoelectric element and an LR circuit. 
(a)

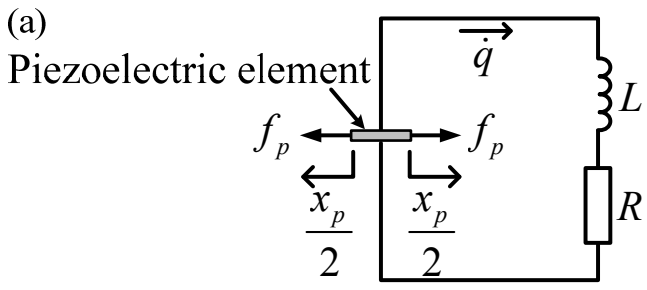

(b)

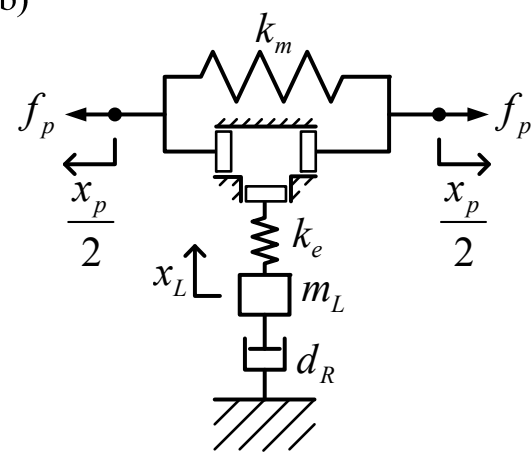

Fig. 3 Analytical model of a piezoelectric element and an LR circuit and its equivalent mechanical model.

$$
V_{p}=-L \ddot{q}-R \dot{q} .
$$

From Eqs. (7), (8), and (17) the equivalent mechanical model of the piezoelectric element and electrical circuit is drawn as Fig. 3(b) (Yamada et al., 2010). Here,

$$
m_{L}=L \theta_{p}^{2}, d_{R}=R \theta_{p}^{2}, k_{e}=\frac{\theta_{p}^{2}}{C_{p}^{S}}, x_{L}=\frac{q}{\theta_{p}} .
$$

The characteristics of the cylinder, pistons, and medium in the cylinder are the same as those in Fig. 1(b). When a resistive circuit is coupled to the piezoelectric element, the mass $m_{L}$ should be zero.

Because the fundamental vibration mode of the beam is usually used in the energy harvesting, the other vibration modes are neglected in this research. Under this condition, the deflection of the beam is expressed as

$$
w_{b}(y, t)=W_{b}(y) \xi_{b}(t),
$$

where $w_{b}$ is the deflection of the beam, $W_{b}$ is the eigenfunction of the deflection, $\xi_{b}$ is the modal displacement, $y$ is the coordinate, and $t$ is the time. The $y$-axis represents the longitudinal direction of the beam, and the left end represents its origin. In cases where multiple vibration modes of the beam are used, the summation of the deflections of the relevant vibration modes should be used instead of Eq. (22). In addition, the eigenfunction of the deflection $W_{b}(y)$ should be derived considering the effect of the piezoelectric element to maintain the accuracy (Yamada et al., 2012). Using the equivalent mechanical model shown in Fig. 3(b) instead of the piezoelectric element and series LR circuit shown in Fig. 2(a), the bending moment that the piezoelectric element applies to the beam is given as

$$
m_{p}=\frac{1}{2} t_{b} f_{p}=\frac{1}{2} t_{b}\left[k_{e}\left(x_{L}-x_{p}\right)-k_{m} x_{p}\right]=\frac{1}{2} t_{b} k_{e} x_{L}-\left(\frac{1}{2} t_{b}\right)^{2}\left(k_{m}+k_{e}\right)\left(\left.\frac{\mathrm{d} W_{b}}{\mathrm{~d} y}\right|_{y=y_{R}}-\left.\frac{\mathrm{d} W_{b}}{\mathrm{~d} y}\right|_{y=y_{L}}\right) \xi_{b},
$$

where $t_{b}$ is the thickness of the beam, and $y_{R}$ and $y_{L}$ are the coordinates of the right and left endpoints of the piezoelectric element in Fig. 2(a), respectively. The neutral axis of the beam was assumed to be identical to the centerline. In addition, the effect of the thickness of the piezoelectric element was approximately ignored here because the bending moment is determined by the deformation of the piezoelectric element in the longitudinal direction. The equation of motion of the beam is expressed as

$$
\rho_{b} A_{b} \frac{\partial^{2} w_{b}}{\partial t^{2}}+E_{b} I_{b} \frac{\partial^{4} w_{b}}{\partial y^{4}}=-m_{b}\left(W_{b}\left(l_{b}\right) \ddot{\xi}_{b}+\ddot{x}_{0}\right) \delta\left(y-l_{b}\right)-\rho_{b} A_{b} \ddot{x}_{0}+m_{p}\left(\frac{\mathrm{d} \delta\left(y-y_{L}\right)}{\mathrm{d} y}-\frac{\mathrm{d} \delta\left(y-y_{R}\right)}{\mathrm{d} y}\right),
$$

where $\rho_{b}, A_{b}, E_{b}, I_{b}$, and $l_{b}$ are the density, cross-sectional area, Young's modulus, second moment of area, and length of the beam, respectively, $m_{b}$ is the mass of the mass point at the right end of the beam, and $\delta$ is the Dirac delta function. Note that Eq. (24) is considered from the viewpoint that moves with $x_{0}$. For this reason, the terms due to the inertial force of the concentrated mass at the right end and distributed mass of the beam are required in the right-hand end of Eq. (24). The third term of the right-hand end of Eq. (24) is the term due to the bending moment applied by the piezoelectric element. The equation of motion with respect to the equivalent mechanical model of the 
electrical system shown in Fig. 3(b) is given as

$$
\begin{aligned}
& m_{L} \ddot{x}_{L}+d_{R} \dot{x}_{L}+k_{e}\left(x_{L}-\theta_{k} \xi_{b}\right)=0, \\
& \theta_{k}=\frac{1}{2} t_{b}\left(\left.\frac{\mathrm{d} W_{b}}{\mathrm{~d} y}\right|_{y=y_{R}}-\left.\frac{\mathrm{d} W_{b}}{\mathrm{~d} y}\right|_{y=y_{L}}\right) .
\end{aligned}
$$

Note that $x_{p}=\theta_{k} \xi_{b}$ because the piezoelectric element is bonded on the beam. From Eqs. (22)-(24), the equation of motion using the modal coordinate system is derived as

$$
\begin{aligned}
& \left(M_{b 1}+M_{b 2}\right) \ddot{\xi}_{b}+D_{b} \dot{\xi}_{b}+\left(K_{b}+k_{m} \theta_{k}^{2}+k_{e} \theta_{k}^{2}\right) \xi_{b}-k_{e} \theta_{k} x_{L}=-B_{r} \ddot{x}_{0}, \\
& M_{b 1}=\rho_{b} A_{b} \int_{0}^{l_{b}} W_{b}^{2} \mathrm{~d} y, M_{b 2}=m_{b}\left(W_{b}\left(l_{b}\right)\right)^{2}, K_{b}=E_{b} I_{b} \int_{0}^{l_{b}} \frac{\mathrm{d}^{4} W_{b}}{\mathrm{~d} y^{4}} W_{b} \mathrm{~d} y, B_{r}=\rho_{b} A_{b} \int_{0}^{l_{b}} W_{b} \mathrm{~d} y+m_{b} W_{b}\left(l_{b}\right),
\end{aligned}
$$

where $D_{b}$ is the modal damping coefficient of the beam. Equation (24) does not have the damping term; however, the damping of the beam was considered in Eq. (27). The reason is that the damping of the additional vibration system is critical to determining the optimum values of the electrical circuit in the case of energy harvesting. To derive the equivalent mechanical model of the whole system, the following displacements are used.

$$
x_{1}=x_{r}+x_{0}, \quad x_{2}=\frac{x_{L}}{\theta_{k} \theta_{m}}, \quad x_{r}=\frac{\xi_{b}}{\theta_{m}}, \quad \theta_{m}=\frac{B_{r}}{M_{b 1}+M_{b 2}} .
$$

From Eqs. (25), (27), and (32)-(35), the equations of motion are transformed into the following equations.

$$
\begin{aligned}
& m_{1} \ddot{x}_{1}+d_{1}\left(\dot{x}_{1}-\dot{x}_{0}\right)+k_{1}\left(x_{1}-x_{0}\right)+k_{2}\left(x_{1}-x_{0}-x_{2}\right)=0, \\
& m_{2} \ddot{x}_{2}+d_{2} \dot{x}_{2}+k_{2}\left(x_{2}-x_{1}+x_{0}\right)=0, \\
& m_{1}=\left(M_{b 1}+M_{b 2}\right) \theta_{m}^{2}, d_{1}=D_{b} \theta_{m}^{2}, k_{1}=\left(K_{b}+k_{m} \theta_{k}^{2}\right) \theta_{m}^{2}, k_{2}=k_{e} \theta_{k}^{2} \theta_{m}^{2}, m_{2}=m_{L} \theta_{k}^{2} \theta_{m}^{2}, d_{2}=d_{R} \theta_{k}^{2} \theta_{m}^{2} .
\end{aligned}
$$

From Eqs. (36) and (37), the equivalent mechanical model of the whole system can be drawn as Fig. 2(b). The characteristics of the cylinder, pistons, and medium in the cylinder are the same as those in Fig. 1(b). When a resistive circuit is coupled to the piezoelectric element, the mass $m_{2}$ should be zero. Note that the same symbols used in Section 2.1 are used for some of the parameters in this model; however, they have no relationship with one another. The equivalent mechanical model of the whole system indicates that there is no essential difference between the energy harvester using the bending vibration and that using the longitudinal vibration of stack-type piezoelectric elements (Renno et al., 2009, Adhikari et al., 2009). Therefore, the knowledge obtained from the previous studies using the longitudinal vibration of stack-type piezoelectric elements can be utilized even when the combination of the bending vibration of a beam and plate-type piezoelectric elements are used. The consumed power of the dashpot whose damping coefficient is $d_{2}$ corresponds to the harvested power.

\section{Optimum values for cases of single excitation frequency}

The objective is to maximize the harvested power, and to derive the optimum values of the electrical circuit and additional vibration system that maximize the harvested power. When the vibration source has only a single excitation frequency, the optimum values of the additional vibration system and electrical circuit can be derived based on impedance matching (Kong et al., 2010). The load mechanical impedance must be the complex conjugate of the source mechanical impedance to satisfy the impedance matching condition. In this research, the equivalent mechanical models are respectively separated into two parts to consider the load and source mechanical impedances as shown in Fig. 4(a) and (b). The load and source mechanical impedances should be considered at the points of the red and blue arrows, respectively. From Fig. 4(a) and (b), both load mechanical impedances are $d_{2}$. Therefore, the source mechanical impedance should be equal to $d_{2}$ to maximize the harvested energy. Because the source mechanical impedances must be a real number $d_{2}$, the resonance of the source side is used in this case. When the impedance matching condition is satisfied, the half of the energy transferred from the vibration source to the additional vibration system is consumed in the dashpot that corresponds to the resistor, and the other half is consumed in the dashpot of the additional vibration system. Optimum values of the additional vibration system and electrical circuit are well known except for the case of 
(a)

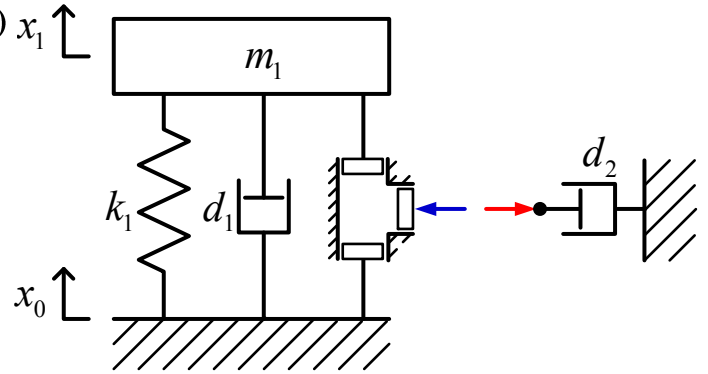

(b) $x_{1}$

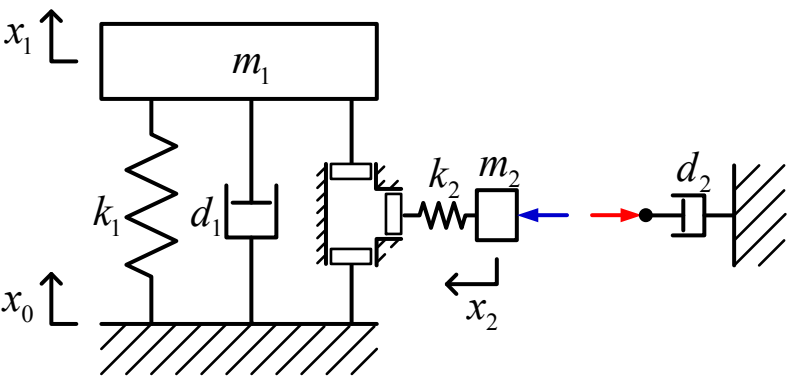

Fig. 4 Separation of the equivalent mechanical models to consider the load and source mechanical impedances.

the combination of a piezoelectric element and a series LR circuit. However, the optimum values for the other two cases are described briefly in this paper for comparison purpose.

\subsection{Impedance matching for energy harvesting using electromagnetic induction}

The nondimensional source mechanical impedance is expressed as

$$
\begin{aligned}
& \frac{Z_{S}}{\sqrt{m_{1} k_{1}}}=\mathrm{j} g+2 \zeta_{1}+\frac{1}{\mathrm{j} g} \\
& g=\frac{\omega}{\Omega_{1}}, \quad \Omega_{1}=\sqrt{\frac{k_{1}}{m_{1}}}, \quad \zeta_{1}=\frac{d_{1}}{2 \sqrt{m_{1} k_{1}}},
\end{aligned}
$$

where $Z_{S}=\mathrm{j} \omega m_{1}+d_{1}+k_{1} /(\mathrm{j} \omega)$ is the source mechanical impedance, and $\omega$ is the excitation angular frequency. The damping ratio of the dashpot that corresponds to the resistor is defined as

$$
\zeta_{1 R}=\frac{d_{2}}{2 \sqrt{m_{1} k_{1}}} .
$$

The nondimensional load mechanical impedance is $Z_{L} / \sqrt{m_{1} k_{1}}=2 \zeta_{1 R}$, where $Z_{L}=d_{2}$ is the load mechanical impedance. To satisfy the mechanical impedance matching condition, the following equation must be satisfied.

$$
\mathrm{j} g+2 \zeta_{1}+\frac{1}{\mathrm{j} g}=2 \zeta_{1 R}
$$

From Eq. (49), the optimum values of the additional vibration system and electrical circuit can be derived as

$$
g=1, \zeta_{1 R}=\zeta_{1},
$$

respectively. Under the condition that the damping ratio $\zeta_{1}$ is constant, $m_{1}$ or $k_{1}$ can be determined arbitrarily. If the value of the resistance cannot be tuned arbitrarily, the resistance must be converted by using an electrical transformer.

\subsection{Impedance matching for energy harvesting using piezoelectric effect}

Similar to Section 3.1, the optimum values of the additional vibration system and electrical circuit can be derived based on impedance matching. In the case of a series LR circuit, the nondimensional source mechanical impedance is expressed as

$$
\begin{aligned}
& \frac{Z_{S}}{\sqrt{m_{2} k_{2}}}=\frac{\mathrm{j} g}{f_{2}}+\frac{f_{2}}{\mathrm{j} g} \frac{-g^{2}+2 \mathrm{j} \zeta_{1} g+1}{-g^{2}+2 \mathrm{j} \zeta_{1} g+1+\beta}, \\
& f_{2}=\frac{\Omega_{2}}{\Omega_{1}}, \quad \Omega_{2}=\sqrt{\frac{k_{2}}{m_{2}}}=\frac{1}{\sqrt{L C_{p}^{S}}}, \quad \beta=\frac{k_{2}}{k_{1}}=\frac{\left(\theta_{p} \theta_{k} \theta_{m}\right)^{2}}{k_{1} C_{p}^{S}},
\end{aligned}
$$

where the same symbols as used in Section 3.1 are used, and $\beta$ is the equivalent stiffness ratio (Yamada et al., 2012). 
The equivalent stiffness ratio $\beta$ is equal to the ratio of the electrical energy stored in the piezoelectric element to the sum of the mechanical energies stored in the additional vibration system and piezoelectric element, under the condition that the electrodes of the piezoelectric element are open-circuited. The damping ratio of the dashpot that corresponds to the resistor is defined as

$$
\zeta_{2 R}=\frac{d_{2}}{2 \sqrt{m_{2} k_{2}}}=\frac{R}{2} \sqrt{\frac{C_{p}^{S}}{L}} .
$$

The nondimensional load mechanical impedance is $Z_{L} / \sqrt{m_{2} k_{2}}=2 \zeta_{2 R}$. To satisfy the mechanical impedance matching condition, the following equation must be satisfied.

$$
\frac{\mathrm{j} g}{f_{2}}+\frac{f_{2}}{\mathrm{j} g} \frac{-g^{2}+2 \mathrm{j} \zeta_{1} g+1}{-g^{2}+2 \mathrm{j} \zeta_{1} g+1+\beta}=2 \zeta_{2 R}
$$

From Eq. (57), the optimum values are given as

$$
\begin{aligned}
& f_{2}=g \sqrt{\frac{\left(-g^{2}+1+\beta\right)^{2}+4 \zeta_{1}^{2} g^{2}}{\left(-g^{2}+1\right)\left(-g^{2}+1+\beta\right)+4 \zeta_{1}^{2} g^{2}}}, \\
& \zeta_{2 R}=\frac{\beta \zeta_{1} g}{\sqrt{\left[\left(-g^{2}+1+\beta\right)^{2}+4 \zeta_{1}^{2} g^{2}\right]\left[\left(-g^{2}+1\right)\left(-g^{2}+1+\beta\right)+4 \zeta_{1}^{2} g^{2}\right]}} .
\end{aligned}
$$

In the case of a series LR circuit, the value of either $f_{2}, \zeta_{2 R}$, or $g$ can be determined arbitrarily. Then, the values of the other two variables are determined using Eqs. (58) and (59). From Eqs. (58) and (59), $g$ must be determined so that $f_{2}$ and $\zeta_{2 R}$ become real numbers. In terms of robustness against the perturbation of the excitation frequency ratio $g$, the slope of $\operatorname{Re}\left(Z_{S}\right)$ should be zero at the selected value of $g$. For this reason, $g$ should be given as

$$
g=\sqrt{1+\beta-2 \zeta_{1}^{2}} .
$$

If Eq. (60) is used, $f_{2}$ and $\zeta_{2 R}$ are determined automatically.

If a resistive circuit is used, the nondimensional source mechanical impedance is expressed as

$$
\frac{Z_{S}}{\frac{k_{2}}{\Omega_{1}}}=\frac{1}{\mathrm{j} g} \frac{-g^{2}+2 \mathrm{j} \zeta_{1} g+1}{-g^{2}+2 \mathrm{j} \zeta_{1} g+1+\beta}
$$

The loss tangent of the dashpot that corresponds to the resistor is defined as

$$
\delta_{2 R}=\frac{d_{2} \Omega_{1}}{k_{2}}=R C_{p}^{S} \Omega_{1}
$$

The nondimensional load mechanical impedance is $Z_{L} /\left(k_{2} / \Omega_{1}\right)=\delta_{2 R}$. To satisfy the mechanical impedance matching condition, the following equation must be satisfied:

$$
\frac{1}{\mathrm{j} g} \frac{-g^{2}+2 \mathrm{j} \zeta_{1} g+1}{-g^{2}+2 \mathrm{j} \zeta_{1} g+1+\beta}=\delta_{2 R}
$$

From Eq. (63), the optimum values are given as

$$
g=\sqrt{\frac{2+\beta-4 \zeta_{1}^{2} \pm \sqrt{\left(\beta-4 \zeta_{1}^{2}\right)^{2}-16 \zeta_{1}^{2}}}{2}}, \quad \delta_{2 R}=\frac{4 \zeta_{1}}{\beta+4 \zeta_{1}^{2} \mp \sqrt{\left(\beta-4 \zeta_{1}^{2}\right)^{2}-16 \zeta_{1}^{2}}} .
$$

In the case of a resistive circuit, there are two kinds of optimum values (Renno et al., 2009). If the optimum values are not real number, the impedance matching condition cannot be satisfied in practice. Therefore, $\beta>4 \zeta_{1}\left(1+\zeta_{1}\right)$ must be satisfied. 


\section{Optimum values for cases of random excitation using white noise}

The objective is to maximize the harvested power under the condition that the energy harvester is subjected to random excitation using white noise. The optimum values of the electrical circuit and additional vibration system for the case of random excitation using white noise have been derived except for the case of the combination of a piezoelectric element and a series LR circuit (Adhikari et al., 2009, Tang and Zuo, 2012). In these derivation procedures, one or two equations are obtained under the condition that maximizes the expectation value of the normalized harvested power, and the optimum values are derived as the analytical solution of the equations. However, the same procedure does not seem to give the optimum values in the case of a series LR circuit because the two equations to be solved are not simple enough to give the exact analytical solutions. Therefore, in this research, the suboptimum values of the series LR circuit and additional vibration system are derived using the different concept.

Parseval's theorem indicates that the time integral of the consumed power of the resistor is equal to the integral of the consumed power of the resistor in the entire frequency. Therefore, if the area of the consumed power of the resistor is maximized in the frequency domain, the time integral of the consumed power of the resistor is also maximized. In addition, the frequency response function of the consumed power in the case of random excitation using white noise is a constant multiple of the frequency response function of the consumed power in the case of impulse excitation. Accordingly, the maximization problem of the harvested energy for the case of random excitation using white noise can be replaced with the maximization problem of the harvested energy from impulse excitation. In this research, the optimum values of the additional vibration system and electrical circuit are derived from this concept. To verify the effectiveness of this concept, the case of using the electromagnetic induction and the case of using a piezoelectric element and a resistive circuit are additionally described similarly to Section 3, and the suboptimum values derived based on this concept are compared to the exact optimum values.

\subsection{Optimum values for cases of random excitation using electromagnetic induction}

In the case where the acceleration $\ddot{x}_{0}$ is given by an impulse function, the energy that is given to the additional vibration system by the impulse excitation is consumed in the dashpot of the additional vibration system and dashpot that corresponds to the resistor in the equivalent mechanical model shown in Fig. 1(b). Because the rate of the consumed energy in the dashpot that corresponds to the resistor is $d_{2} /\left(d_{1}+d_{2}\right)=\zeta_{1 R} /\left(\zeta_{1}+\zeta_{1 R}\right)$, the harvested energy increases with the increase in the damping ratio of the electrical system $\zeta_{1 R}$. From Eqs. (4) and (48), the optimum resistance is zero. This fact does not make practical sense; however, this fact indicates that the damping effect of the electrical system on the additional vibration system should be maximized to maximize harvested energy. This result is completely consistent with the exact analytical solution (Tang and Zuo, 2012). Naturally, this concept is effective for all cases where the vibration system experiences free vibration. In the case where the acceleration $\ddot{x}_{0}$ is given by an impulse function, the displacement $x_{0}$ is given as a ramp function. This motion is not realistic; however, this impulse excitation is considered as a tool to derive the concept to maximize harvested energy in the case of random excitation.

\subsection{Optimum values for cases of random excitation using piezoelectric effect}

The equivalent mechanical model shown in Fig. 2(b) indicates that the part of the electrical system works as a vibration absorber. Similar to Section 4.1, the damping effect of the vibration absorber on the additional vibration system should be maximized to maximize the harvested energy. The knowledge obtained from the studies of the passive vibration suppression using a piezoelectric element and a series LR circuit can be utilized when the part of the electrical system is regarded as a vibration absorber. In the case of a series LR circuit, the suboptimum natural frequency ratio between the electrical system and additional vibration system is expressed as

$$
f_{2}=(1+\beta) \sqrt{\frac{2}{2+\beta}} .
$$

Equation (66) is the optimum natural frequency ratio derived using two fixed points method when the additional vibration system is excited by the external force, and mobility is evaluated in passive vibration suppression (Yamada et al., 2010). Equation (66) can be used for energy harvesting as the suboptimum value because the mobility is defined as 
the reciprocal of the mechanical impedance, and the mechanical impedance is the broad definition of the damping. Note that Eq. (66) is derived under the condition that the damping ratio of the additional vibration system $\zeta_{1}$ is negligible. Because the damping effect of the vibration absorber is important here, the existence of $\zeta_{1}$ has little effect on the optimum natural frequency ratio. To be exact, the additional vibration system is excited by the acceleration $\ddot{x}_{0}$ rather than the external force in this research. However, base excitation can be replaced with external force excitation if the equation of motion is considered from the viewpoint that moves with $x_{0}$. The optimum resistance ratio for passive vibration suppression has been derived using the two fixed points method (Yamada et al., 2010). However, this optimum resistance ratio is not optimum for energy harvesting because the value minimizes the maximum value of the magnitude of the mobility, and the value does not maximize the damping effect of the vibration absorber on the additional vibration system.

The mechanical impedance that is given to the additional vibration system by the vibration absorber must be considered to maximize the damping effect of the vibration absorber. Here, the mechanical impedance of the vibration absorber must be derived at the point of the green arrow in Fig. 5. Equation (66) reduces the absolute value of the imaginary part of the mechanical impedance $\operatorname{Im}\left(Z_{V A}\right)$ around the natural frequency of the additional vibration system, where $Z_{V A}$ is the mechanical impedance of the vibration absorber. In the frequency region where the absolute value of the imaginary part of the mechanical impedance $Z_{V A}$ is small in value, the real part of the mechanical impedance $\operatorname{Re}\left(Z_{V A}\right)$ should be broadly large to increase the amount of harvested energy. Based on this concept, the suboptimum resistance ratio for the energy harvesting can be derived by the condition that the slope of the real part of the mechanical impedance $\operatorname{Re}\left(Z_{V A}\right)$ is zero at the excitation frequency ratio $g_{C}$, which is defined as the geometric mean of the excitation frequency ratios of the two fixed points (Yamada et al., 2010). The excitation frequency ratio $g_{C}$ is expressed as

$$
g_{C}=\sqrt{1+\beta} .
$$

The suboptimum resistance ratio is given as

$$
\zeta_{2 R}=\frac{1}{2} \sqrt{\frac{\beta}{1+\beta}} .
$$

The suboptimum resistance ratio for energy harvesting is about $(2 / 3)^{1 / 2}$ times the optimum resistance ratio for passive vibration suppression using mobility for evaluation (Yamada et al., 2010). Because this concept and Eqs. (66) and (68) are not guaranteed to be effective, the effectiveness is verified through numerical calculations in Section 6.

In cases where energy is harvested using a resistive circuit, the optimum loss tangent has been derived by Adhikari et al. (2009). The optimum loss tangent is expressed as

$$
\delta_{2 R}=\sqrt{\frac{1}{1+\beta}} .
$$

Here, the suboptimum loss tangent is derived based on the concept of this research, and compared to the exact optimum loss tangent. The optimum loss tangent for passive vibration suppression that has been derived using the mobility and a fixed point method can be used as the suboptimum loss tangent for energy harvesting. The suboptimum loss tangent is expressed as

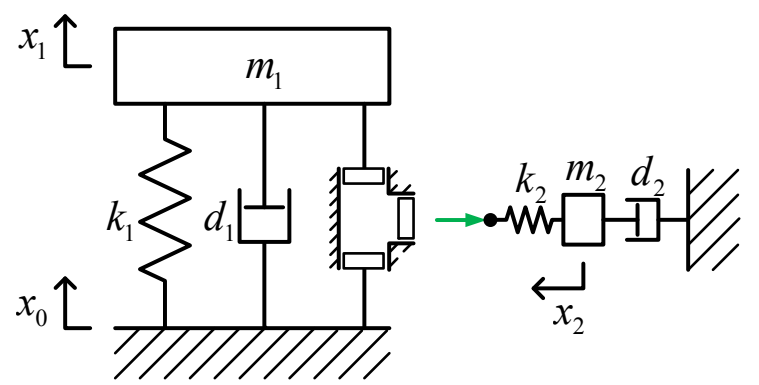

Fig. 5 Separated model to consider the mechanical impedance of the vibration absorber that corresponds to the electrical system. 


$$
\delta_{2 R}=\sqrt{\frac{2(4+\beta)}{(2+\beta)(4+3 \beta)}} .
$$

Because the damping effect of the electrical system suppresses the vibration of the host structure in the case of a resistive circuit, the optimum loss tangent for passive vibration suppression is approximately equal to that for energy harvesting. Note that Eq. (70) is derived under the condition that the damping ratio of the additional vibration system $\zeta_{1}$ is negligible, similar to the case of the series LR circuit. Because both optimum and suboptimum loss tangents given by Eqs. (69) and (70) can be approximated by $1-\beta / 2$ under the condition that the equivalent stiffness ratio $\beta$ is much less than one, the difference between Eqs. (69) and (70) is significantly small. From this perspective, the concept of this research is reasonable.

\section{Harvested energy}

The consumed energy of the resistor corresponds to the harvested energy. The average power of the resistor is evaluated in this research.

\subsection{Average power of resistor using electromagnetic induction}

The average power of the resistor is derived as

$$
P_{R}=\frac{1}{2} \operatorname{Re}\left(d_{2} \mathrm{j} \omega\left(X_{1}-X_{0}\right) \cdot \overline{\mathrm{j} \omega\left(X_{1}-X_{0}\right)}\right)=\frac{m_{1} A_{0}^{2}}{\Omega_{1}} \frac{\zeta_{1 R} g^{2}}{\left(-g^{2}+1\right)^{2}+4\left(\zeta_{1}+\zeta_{1 R}\right)^{2} g^{2}},
$$

where $A_{0}$ is the amplitude of the acceleration $\ddot{x}_{0}$. From Eqs. (50), (51) and (71), in the case of a single excitation frequency, the average power of the resistor is inversely proportional to the damping ratio $\zeta_{1}$ when the optimum values are used. Furthermore, the harvested energy increases with the increase in $m_{1} A_{0}^{2} / \Omega_{1}$. In the following sections, the nondimensional average power is used for generalization. The nondimensional average power is defined as

$$
\bar{P}_{R}=\frac{P_{R}}{\frac{m_{1} A_{0}^{2}}{\Omega_{1}}}=\frac{\zeta_{1 R} g^{2}}{\left(-g^{2}+1\right)^{2}+4\left(\zeta_{1}+\zeta_{1 R}\right)^{2} g^{2}}
$$

The average power and nondimensional average power of the damping of the additional vibration system are respectively given as

$$
\begin{aligned}
& P_{d}=\frac{1}{2} \operatorname{Re}\left(d_{1} \mathrm{j} \omega\left(X_{1}-X_{0}\right) \cdot \overline{\mathrm{j} \omega\left(X_{1}-X_{0}\right)}\right)=\frac{m_{1} A_{0}^{2}}{\Omega_{1}} \frac{\zeta_{1} g^{2}}{\left(-g^{2}+1\right)^{2}+4\left(\zeta_{1}+\zeta_{1 R}\right)^{2} g^{2}}, \\
& \bar{P}_{d}=\frac{P_{d}}{\frac{m_{1} A_{0}^{2}}{\Omega_{1}}}=\frac{\zeta_{1} g^{2}}{\left(-g^{2}+1\right)^{2}+4\left(\zeta_{1}+\zeta_{1 R}\right)^{2} g^{2}} .
\end{aligned}
$$

\subsection{Average power of resistor using piezoelectric effect}

In the case of a series LR circuit, the nondimensional average powers of the resistor and additional vibration system are respectively given as

$$
\bar{P}_{R}=\frac{P_{R}}{\frac{m_{1} A_{0}^{2}}{\Omega_{1}}}=\frac{\zeta_{2 R} \beta g^{2}}{f_{2}}\left|\frac{f_{2}^{2}}{-g^{2}+2 \mathrm{j} \zeta_{2 R} f_{2} g+f_{2}^{2}} \frac{1}{-g^{2}+2 \mathrm{j} \zeta_{1} g+1+\beta \frac{-g^{2}+2 \mathrm{j} \zeta_{2 R} f_{2} g}{-g^{2}+2 \mathrm{j} \zeta_{2 R} f_{2} g+f_{2}^{2}}}\right|^{2},
$$




$$
\bar{P}_{d}=\frac{P_{d}}{\frac{m_{1} A_{0}^{2}}{\Omega_{1}}}=\zeta_{1} g^{2}\left|\frac{1}{-g^{2}+2 \mathrm{j} \zeta_{1} g+1+\beta \frac{-g^{2}+2 \mathrm{j} \zeta_{2 R} f_{2} g}{-g^{2}+2 \mathrm{j} \zeta_{2 R} f_{2} g+f_{2}^{2}}}\right|^{2}
$$

In the case of a resistive circuit, the nondimensional average powers of the resistor and additional vibration system are respectively expressed as

$$
\begin{aligned}
& \bar{P}_{R}=\frac{P_{R}}{\frac{m_{1} A_{0}^{2}}{\Omega_{1}}}=\frac{\delta_{2 R} \beta g^{2}}{2}\left|\frac{1}{1+\mathrm{j} \delta_{2 R} g} \frac{1}{-g^{2}+2 \mathrm{j} \zeta_{1} g+1+\beta \frac{\mathrm{j} \delta_{2 R} g}{1+\mathrm{j} \delta_{2 R} g}}\right|^{2}, \\
& \bar{P}_{d}=\frac{P_{d}}{\frac{m_{1} A_{0}^{2}}{\Omega_{1}}}=\zeta_{1} g^{2}\left|\frac{1}{-g^{2}+2 \mathrm{j} \zeta_{1} g+1+\beta \frac{\mathrm{j} \delta_{2 R} g}{1+\mathrm{j} \delta_{2 R} g}}\right|^{2}
\end{aligned}
$$

From Eqs. (75) and (77), the amount of harvested energy increases with the increase in $m_{1} A_{0}^{2} / \Omega_{1}$.

\section{Numerical calculation}

When vibration sources include only a single excitation frequency, the optimum values of the additional vibration system and electrical circuit can be derived based on impedance matching as described in Section 3. In this case, the validation using numerical calculations is not necessary because the optimum values based on the impedance matching are exact in principle. The harvestable energy can be estimated using the average power formulated in Section 5. When the optimum values are used, the nondimensional average powers are given as

$$
\bar{P}_{R}=\bar{P}_{d}=\frac{1}{16 \zeta_{1}}
$$

Equation (79) is true regardless of the use of electromagnetic induction or piezoelectric effect and the types of electrical circuits. Equation (79) can be easily derived in the case of electromagnetic induction (Stephen, 2006). In this research, the mechanical impedances of the source and load were considered by separating the equivalent mechanical model of the whole system as shown in Fig. 4. However, the dividing point can be selected arbitrarily in principle. Even when the connecting point between the dashpot of the additional vibration system and the rest was adopted as the dividing point, the mechanical impedance of the dashpot of the additional vibration system is a complex conjugate of the mechanical impedance of the rest. This fact indicates that the amounts of the harvested energy are equal regardless of the three methods. The half of the energy that is transferred from the vibration source to the additional vibration system is harvested by the resistor, and the other half is wasted in the dashpot of the additional vibration system. The amount of harvested energy is independent of the value of the torque constant $K_{T E}$ or equivalent stiffness ratio $\beta$ when the impedance matching condition is satisfied. The numerical calculation results of the nondimensional average power $\bar{P}_{R}$ using a piezoelectric element, a series LR circuit, and different targeted frequency ratios are shown in Fig. 6. Here, $g_{a}$ is the targeted frequency ratio, and $\zeta_{1}=0.01$ and $\beta=0.01$ were used. The optimum natural frequency ratio and optimum resistance ratio were derived substituting $g=g_{a}$ into Eqs. (58) and (59), respectively. The nondimensional average power represents the maximum value given by Eq. (79) at the targeted frequency ratios in these numerical calculations. In addition, the targeted frequency ratio given by Eq. (60) indicates the high robustness against the perturbation of the targeted excitation frequency ratio $g_{a}$.

The effectiveness of the suboptimum values of the additional vibration system and series LR circuit for the case of random excitation using white noise was verified through the numerical calculations. Parseval's theorem indicates that the area of the average power $S_{P R}$ is available for evaluating the harvested energy.

$$
S_{P R}=\frac{1}{2 \pi} \int_{-\infty}^{\infty} P_{R} \mathrm{~d} \omega=\frac{m_{1} A_{0}^{2}}{2 \pi} \int_{-\infty}^{\infty} \bar{P}_{R} \mathrm{~d} g
$$

In the following numerical calculations, the nondimensional area of the average power $\bar{S}_{P R}$ was evaluated as 


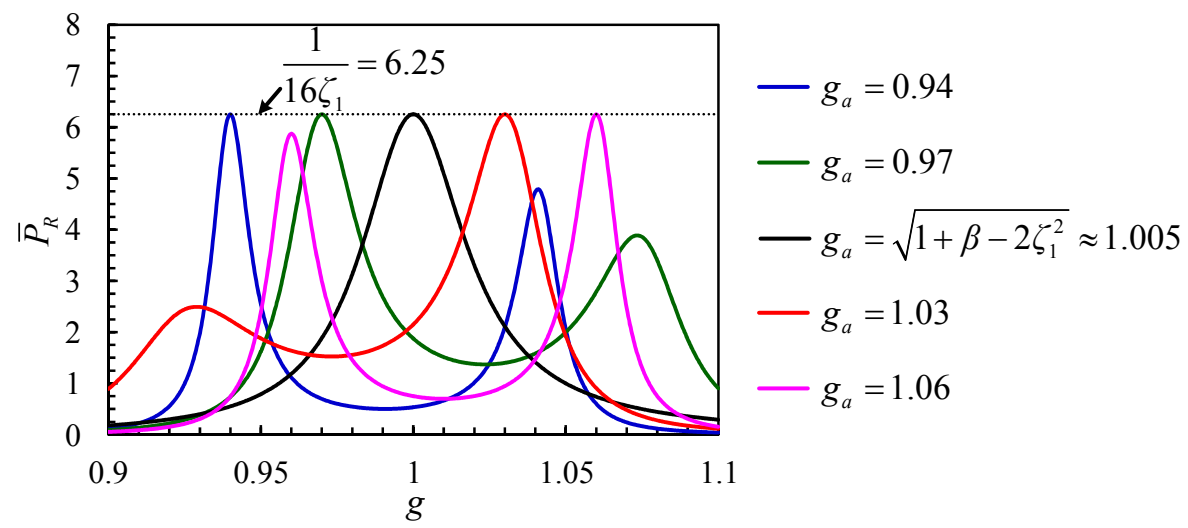

Fig. 6 Numerical calculation results of the nondimensional average power of the energy harvester using a piezoelectric element, a series LR circuit, and different targeted frequency ratios. $\zeta_{1}=0.01$ and $\beta=0.01$ were used.

$$
\bar{S}_{P R}=\frac{S_{P R}}{\frac{m_{1} A_{0}^{2}}{4}}=\frac{2}{\pi} \int_{-\infty}^{\infty} \bar{P}_{R} \mathrm{~d} g
$$

Using this definition of nondimensionalization, the maximum value of $\bar{S}_{P R}$ represents one. In the case of electromagnetic induction, $\bar{S}_{P R}$ is derived as

$$
\bar{S}_{P R}=\frac{2}{\pi} \int_{-\infty}^{\infty} \frac{\zeta_{1 R} g^{2}}{\left(-g^{2}+1\right)^{2}+4\left(\zeta_{1}+\zeta_{1 R}\right)^{2} g^{2}} \mathrm{~d} g=\frac{\zeta_{1 R}}{\zeta_{1}+\zeta_{1 R}} .
$$

In contrast, in the case of using a piezoelectric element and a series LR circuit, $\bar{S}_{P R}$ is expressed as

$$
\bar{S}_{P R}=\frac{\zeta_{2 R} \beta f_{2}^{2}\left(\zeta_{1}+\zeta_{2 R} f_{2}\right)}{\left(\zeta_{1}+\zeta_{2 R} f_{2}\right)\left(\zeta_{1} f_{2}+\zeta_{2 R}+\beta \zeta_{2 R}\right)\left(1+f_{2}^{2}+4 \zeta_{1} \zeta_{2 R} f_{2}+\beta\right)-f_{2}\left(\zeta_{1}+\zeta_{2 R} f_{2}\right)^{2}-f_{2}\left(\zeta_{1} f_{2}+\zeta_{2 R}+\beta \zeta_{2 R}\right)^{2}} .
$$

The procedure of the improper integral was described by Adhikari et al. (2009) and Tang and Zuo (2012). In addition, in the case of using a piezoelectric element and a resistive circuit, $\bar{S}_{P R}$ is given as

$$
\bar{S}_{P R}=\frac{\delta_{2 R} \beta}{2 \zeta_{1} \delta_{2 R}^{2}(1+\beta)+\delta_{2 R}\left(\beta+4 \zeta_{1}^{2}\right)+2 \zeta_{1}} .
$$

When $\zeta_{1}=0, \bar{S}_{P R}$ is equal to one in all cases. Because all the energy is harvested in the resistor under this condition, this is the maximum value of $\bar{S}_{P R}$. If impulse excitation is considered instead of random excitation using white noise, the amounts of the energy transferred from the vibration source to the additional vibration system are equal regardless of the use of electromagnetic induction or piezoelectric effect and the types of electrical circuits. Therefore, the maximum value of $\bar{S}_{P R}$ is one in all cases. Because the damping effect of the electrical system on the additional vibration system should be large in the case of random excitation, the amount of harvested energy increases with the increase in the torque constant $K_{T E}$ or equivalent stiffness ratio $\beta$ (Yamada et al., 2014, 2015). The optimum values described in Section 4 can be derived as the analytical solutions under the condition that $\bar{S}_{P R}$ is maximized. The analytical solutions can be derived from Eqs. (82) and (84); however, the analytical solution cannot be obtained from Eq. (83).

The numerical calculation results of the nondimensional area of the average power $\bar{S}_{P R}$ using electromagnetic induction are shown in Fig. 7. The nondimensional area of the average power $\bar{S}_{P R}$ monotonically increases with an increase in the damping ratio $\zeta_{1 R}$ as described in Section 4.1.

The numerical calculation results of $f_{2}$ and $\zeta_{2 R}$ are shown in Fig. 8. Here, the suboptimum values derived in Section 4.2 and optimum values are compared, and $\zeta_{1}=0.01$ was used. The optimum value was obtained by the numerical optimization. Because the optimum values of $f_{2}$ and $\zeta_{2 R}$ obtained by the numerical optimization are nearly independent of the damping ratio $\zeta_{1}$, only the numerical calculation results using $\zeta_{1}=0.01$ were shown in Fig. 8. From Eqs. (40), (41), and (55), the equivalent stiffness ratio $\beta$ is less than $k_{e} / k_{m}=d_{31}^{2} E_{p} /\left(\varepsilon_{33}^{T}-d_{31}^{2} E_{p}\right)$ 
when a plate-type piezoelectric element is bonded on a beam. $k_{e} / k_{m}$ is usually around 0.17 . Therefore, the difference between the suboptimum and optimum values is significantly small. The optimum resistance ratio for passive vibration suppression is additionally shown in Fig. 8(b) (Yamada et al., 2010). The optimum resistance ratio for energy harvesting is smaller than that for passive vibration suppression as described in Section 4.2. The numerical calculation results of the nondimensional area of the average power $\bar{S}_{P R}$ using a piezoelectric element and a series LR circuit are shown in Fig. 9. Here, the numerical calculation results using the suboptimum and optimum values are compared, and $\zeta_{1}=0.01,0.03$, and 0.05 were used. Because the difference of the nondimensional area of the average power $\bar{S}_{P R}$ between the results using the suboptimum and optimum values is negligible, the derived suboptimum values can be utilized in practice. The nondimensional area of the average powers $\bar{S}_{P R}$ using a parallel LR circuit and a resistive circuit are additionally shown in Fig. 9 (Adhikari et al., 2009). $\bar{S}_{P R}$ using a series LR circuit is slightly larger than $\bar{S}_{P R}$ using a parallel LR circuit. Because the performance of the series LR circuit is slightly higher than that of the parallel LR circuit in passive vibration suppression (Yamada et al., 2010), this numerical calculation results are reasonable. The nondimensional area of the average power $\bar{S}_{P R}$ using a resistive circuit is smaller than those using the LR circuits.



Fig. 7 Numerical calculation results of the nondimensional area of the average power of the energy harvester using electromagnetic induction.
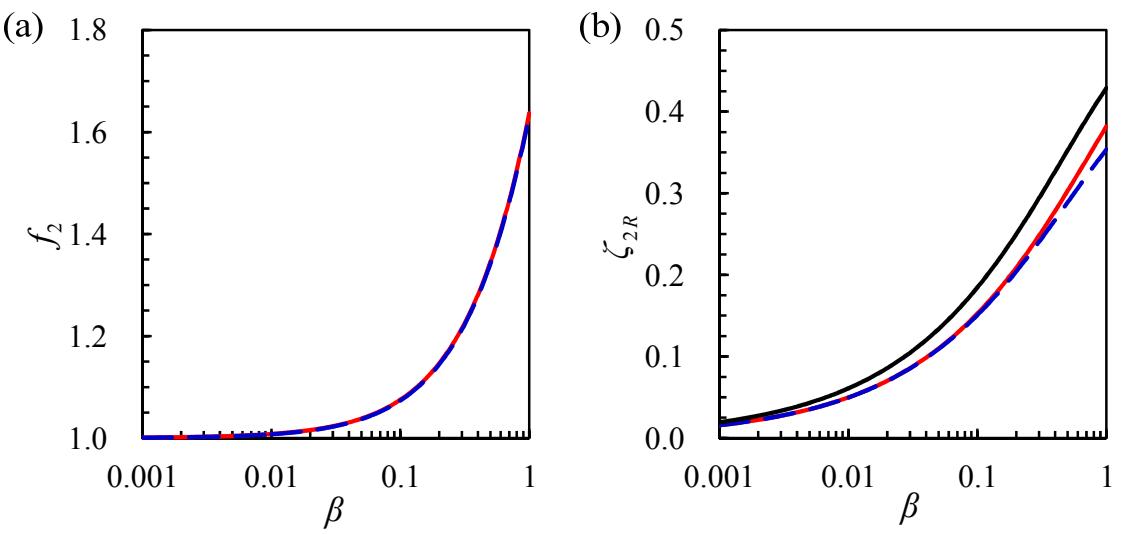

- Suboptimum value Optimum value obtained by numerical optimization Optimum resistance ratio for passive vibration suppression

Fig. 8 Numerical calculation results of the suboptimum and optimum values of the natural frequency ratio $f_{2}$ and resistance ratio $\zeta_{2 R}$ of the energy harvester using a piezoelectric element and a series LR circuit. 



- - Series LR circuit using suboptimum value

- Series LR circuit using optimum value obtained by numerical optimization

.... Parallel LR circuit

Resistive circuit

Fig. 9 Numerical calculation results of the nondimensional area of the average power of the energy harvester using a piezoelectric element.

\section{Conclusion}

In this research, in the energy harvesting methods using a beam, a piezoelectric element, and a series LR circuit, the optimum values of the additional vibration system and series LR circuit were theoretically derived for two cases: one is the case where vibration sources have only a single excitation frequency, and the other is the case of random excitation using white noise. The governing equations were derived using the equivalent mechanical models. The effectiveness of the derived optimum values was verified through the numerical calculations. We used simple analytical models to reach the objective of obtaining essential knowledge in this research. In the investigation for practical use, the internal resistance of DC motors and electrical magnets and the Q factor of actual inductors should be taken into consideration.

\section{Acknowledgment}

This work was supported in part by the Grant-in-Aid for Scientific Research (C) (JSPS, No. 26420187).

\section{References}

Adhikari, S., Friswell, M. I. and Inman, D. J., Piezoelectric energy harvesting from broadband random vibrations, Smart Materials and Structures, Vol. 18, No. 11 (2009), p. 115005 (7pp).

Caliò, R., Rongala, U. B., Camboni, D., Milazzo, M., Stefanini, C., de Petris, G. and Oddo, C. M., Piezoelectric energy harvesting solutions, Sensors, Vol. 14, No. 3 (2014), pp. 4755-4790.

Guyomar, D., Badel, A., Lefeuvre, E. and Richard, C., Toward energy harvesting using active materials and conversion improvement by nonlinear processing, IEEE Transactions on Ultrasonics, Ferroelectrics, and Frequency Control, Vol. 52, No. 4 (2005), pp. 584-595.

Halvorsen, E., Energy harvesters driven by broadband random vibrations, Journal of Microelectromechanical Systems, Vol. 17, No. 5 (2008), pp. 1061-1071.

Hendijanizadeh, M., Design and optimisation of constrained electromagnetic energy harvesting devices, Thesis for the degree of Doctor of Philosophy, University of Southampton, (2014).

Kong, N., Ha, D. S., Erturk, A. and Inman, D. J., Resistive impedance matching circuit for piezoelectric energy harvesting, Journal of Intelligent Material Systems and Structures, Vol. 21, (2010), pp. 1293-1302.

Litak, G., Friswell, M. I. and Adhikari, S., Magnetopiezoelastic energy harvesting driven by random excitations, Applied Physics Letters, Vol. 96, (2010), 214103. 
Masuda, A., Senda, A., Sanada, T. and Sone, A., Global stabilization of high-energy response for a duffing-type wideband nonlinear energy harvester via self-excitation and entrainment, Journal of Intelligent Material Systems and Structures, Vol. 24, (2013), pp. 1598-1612.

Mitcheson, P. D., Green, T. C., Yeatman, E. M. and Holmes, A. S., Architectures for vibration-driven micropower generators, Journal of Microelectromechanical systems, Vol. 13, No. 3 (2004), pp. 429-440.

Mitcheson, P. D., Yeatman, E. M., Rao, G. K., Holmes, A. S. and Green, T. C., Energy harvesting from human and machine motion for wireless electronic devices, Proceedings of the IEEE, Vol. 96, No. 9 (2008), pp. 1457-1486.

Ottman, G. K., Hofmann, H. F., Bhatt, A. C. and Lesieutre, G. A., Adaptive piezoelectric energy harvesting circuit for wireless remote power supply, IEEE Transactions on Power Electronics, Vol. 17, No. 5 (2002), pp. 669-676.

Ottman, G. K., Hofmann, H. F. and Lesieutre, G. A., Optimized piezoelectric energy harvesting circuit using step-down converter in discontinuous conduction mode, IEEE Transactions on Power Electronics, Vol. 18, No. 2 (2003), pp. 696-703.

Renaud, M., Karakaya, K., Sterken, T., Fiorini, P., Hoof, C. V. and Puers, R., Fabrication, modelling and characterization of MEMS piezoelectric vibration harvesters, Sensors and Actuators A, Vol. 145-146, (2008), pp. 380-386.

Renno, J. M., Daqaq, M. F., and Inman, D. J., On the optimal energy harvesting from a vibration source, Journal of Sound and Vibration, Vol. 320, Issue 1-2 (2009), pp. 386-405.

Roundy, S., On the effectiveness of vibration-based energy harvesting, Journal of Intelligent Material Systems and Structures, Vol. 16, (2005), pp. 809-823.

Stephen, N. G., On energy harvesting from ambient vibration, Journal of Sound and Vibration, Vol. 293, (2006), pp. 409-425.

Tang, L., Yang, Y. and Soh C. K., Toward broadband vibration-based energy harvesting, Journal of Intelligent Material Systems and Structures, Vol. 21, (2010), pp. 1867-1897.

Tang, X. and Zuo, L., Vibration energy harvesting from random force and motion excitations, Smart Materials and Structures, Vol. 21, No. 7 (2012), p. 075025 (9pp).

Yamada, K., Matsuhisa, H., Utsuno, H. and Sawada, K., Optimum tuning of series and parallel LR circuits for passive vibration suppression using piezoelectric elements, Journal of Sound and Vibration, Vol. 329, Issue 24 (2010), pp. 5036-5057.

Yamada, K., Matsuhisa, H. and Utsuno, H., A new method for accurately determining the modal equivalent stiffness ratio of bonded piezoelectric structures, Journal of Sound and Vibration, Vol. 331, Issue 14 (2012), pp. 3317-3344.

Yamada, K., Matsuhisa, H. and Utsuno, H., Improvement of efficiency of piezoelectric element attached to beam based on mechanical impedance matching, Journal of Sound and Vibration, Vol. 333, Issue 1 (2014), pp. 52-79.

Yamada, K., Enhancing efficiency of piezoelectric element attached to beam using extended spacers, Journal of Sound and Vibration, Vol. 341, (2015), pp. 31-52.

Yamada, K., Complete passive vibration suppression using multi-layered piezoelectric element, inductor, and resistor, Journal of Sound and Vibration, Vol. 387, (2017), pp. 16-35.

Yamamoto, Y., Yoshimizu, K. and Makihara, K., Synthetic assessment of self-powered energy-harvesting including robustness evaluation, Mechanical Engineering Journal, Vol. 2, No. 5 (2015), pp. 1-12. 